\title{
Hyalinizing Trabecular Tumor of the Thyroid Gland, a Diagnostic Challenge in Fine-Needle Aspiration Cytology: Case Report
}

\author{
Ye-Young Rhee $\cdot$ Hong Kyu Jung ${ }^{1}$ \\ Se Hoon $\mathrm{Kim}^{2}$. Soo Hee Kim \\ Pathology Center, Seegene Medical Foundation, \\ Seoul; ' Department of Surgery and Health Care \\ Center, Seran Hospital, Seoul; ' 2 Department of \\ Pathology, Yonsei University College of Medicine, \\ Severance Hospital, Seoul, Korea \\ Received: March 16, 2018 \\ Revised: April 18, 2018 \\ Accepted: April 27, 2018 \\ Corresponding Author \\ Soo Hee Kim, MD, PhD \\ Pathology Center, Seegene Medical Foundation, \\ 320 Cheonho-daero, Seongdong-gu, Seoul 04805, \\ Korea \\ Tel: +82-2-2218-9346 \\ Fax: +82-2-2218-9310 \\ E-mail:kshpath@gmail.com
}

\begin{abstract}
Hyalinizing trabecular tumor $(\mathrm{HTT})$ is a rare thyroid tumor with low to minimal malignant potential. $\mathrm{HTT}$ is often misinterpreted as other thyroid tumors, including papillary thyroid carcinoma (PTC) and medullary thyroid carcinoma (MTC), on fine-needle aspiration (FNA) cytology, because of its overlapping cytologic features, such as nuclear grooves and intranulcear pseudoinclusions. Although cytopathologists cannot definitely conclude HTT by FNA cytology, suspicion of HTT is necessary to avoid misdiagnosing HTT as PTC or MTC and to avoid unnecessary aggressive treatment. Here, we report a case of HTT with novel cytologic features in CellPrep liquid based cytology that was diagnosed as suspicious for papillary carcinoma by FNA and finally diagnosed as $\mathrm{HTT}$ in the surgical specimen.
\end{abstract}

Key Words: Hyalinizing trabecular tumor; Thyroid gland; Liquid-based cytology
Hyalinizing trabecular tumor (HTT) is an uncommon thyroid tumor that is usually benign and is, often misdiagnosed as another thyroid neoplasm, such as papillary thyroid carcinoma (PTC) or medullary thyroid carcinoma (MTC), by fine-needle aspiration (FNA) cytology, due to its similar nuclear features as PTC or the presence of hyaline material resembling amyloid in MTC. ${ }^{1,2}$ Diagnosis of HTT in FNA specimens is challenging, as reported in Zipkin's first report in 1905, ${ }^{3}$ and Carney's first detailed description in $1987,{ }^{4}$ because HTT has these overlapping cytologic features with PTC and MTC. There are several case reports of HTT and studies dealing with the pathologic features and biologic behavior of HTT, as well as diagnostic tools for accurate diagnosis of HTT. ${ }^{5-7}$ Here, we report a case of HTT that includes several key features to remember for correct diagnosis.

\section{CASE REPORT}

A 63-year-old woman with history of breast cancer underwent a medical checkup. Ultrasound examination revealed one hypoechoic lesion measuring $0.6 \times 0.4 \mathrm{~cm}$ in the left thyroid lobe
(Fig. 1A) and two small cystic lesions in the right thyroid lobe. Liquid based cytology (LBC; CellPrep, Biodyne, Seongnam, Korea) FNA of the left lobe nodule showed features of PTC including overlapping enlarged nuclei, occasional nuclear grooves, and intranuclear pseudoinclusions. The lesion was diagnosed as suspicious for papillary carcinoma. Computed tomography revealed a $0.6 \mathrm{~cm}$ low density nodule with no evidence of extracapsular invasion and no significant lymph node enlargement (Fig. 1B). The surgeon decided to perform left lobectomy based on the assumption that the lesion could be a benign tumor in the final pathologic report, because of uncertain radiologic features.

On gross examination, one well circumscribed yellow to tan firm nodule measuring $0.6 \times 0.5 \times 0.4 \mathrm{~cm}$ was identified (Fig. $2 \mathrm{~A})$. Histologic examination revealed a well circumscribed nodule composed of trabecular and alveolar patterns of tumor cells. Hyalinization was observed between cell trabeculae. Tumor cells had abundant cytoplasm, low nuclear to cytoplasmic ratio, perinuclear clearing, nuclear grooves, and intranuclear pseudoinclusions (Fig. 2B, C)

Immunohistochemistry showed diffuse membrane positive 

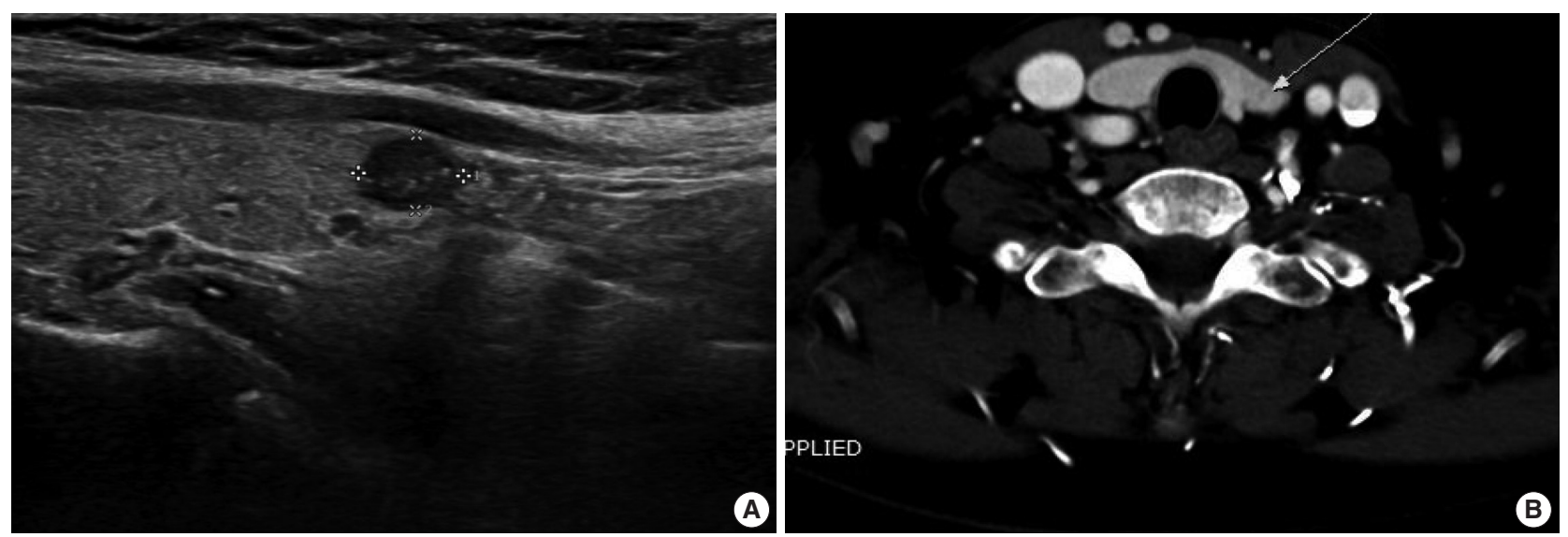

Fig. 1. Radiologic findings. (A) Ultrasonography shows a $0.6 \mathrm{~cm}$ hypoechoic nodule in the left lobe (longitudinal). (B) Computed tomography reveals a $0.6 \mathrm{~cm}$ low density nodule with no evidence of extracapsular invasion and no significant lymph node enlargement.
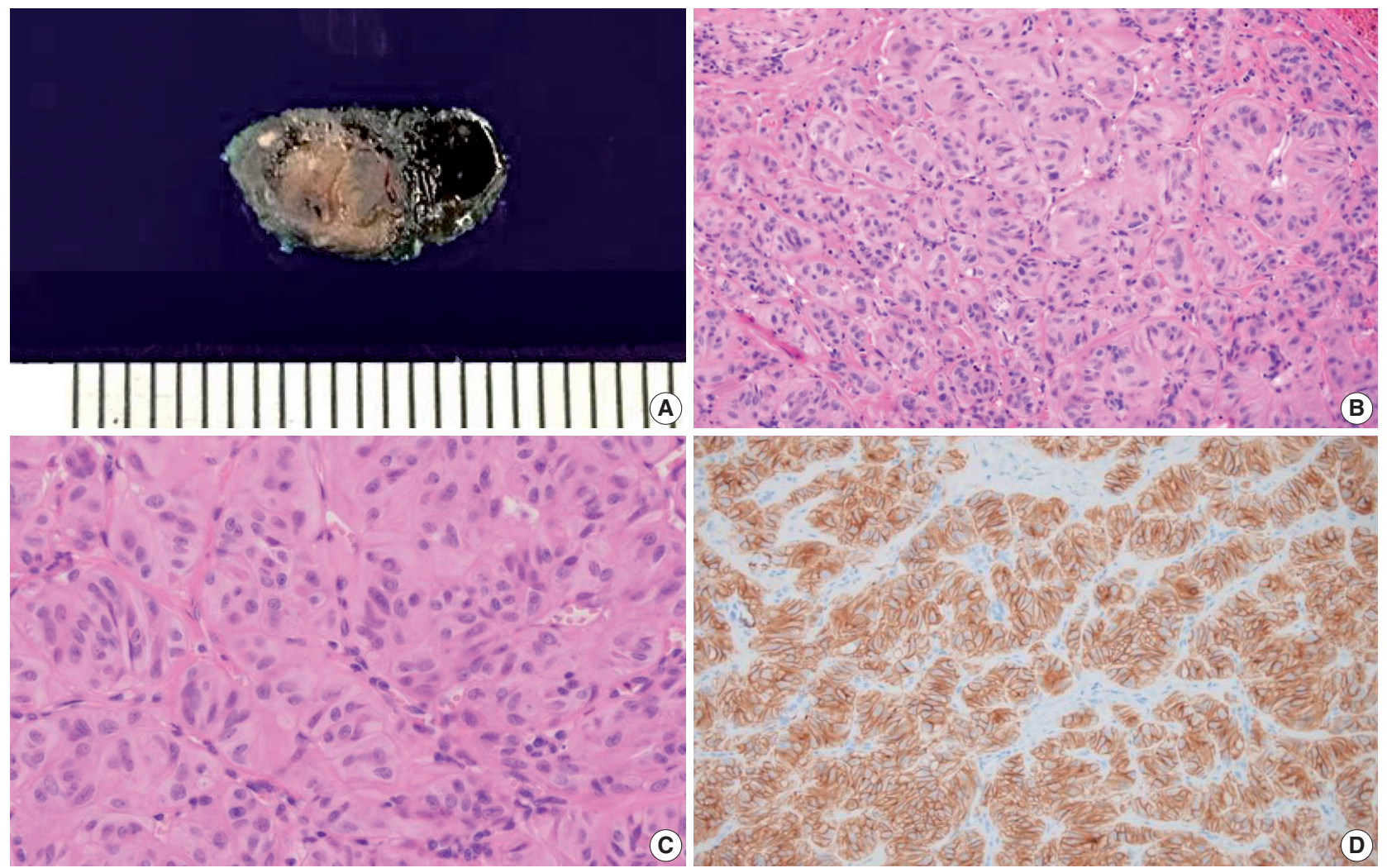

Fig. 2 . Gross specimen, microscopic findings (hematoxylin and eosin), and immunohistochemical staining of Ki-67 in the surgical specimen. (A) Gross specimen showing well circumscribed yellow to tan mass. (B, C) Histologic image shows trabecular pattern with oval to elongated tumor cells and intratrabecular hyalinization. (D) Ki-67 immunostaining using MIB1 monoclonal antibody shows characteristic peripheral membranous and cytoplasmic staining.

reactivity for $\mathrm{Ki}-67$ (MIB1 clone) at room temperature, which is a distinct immunohistochemical characteristic of HTT (Fig. 2D). Immunohistochemical staining also revealed diffuse positivity for CD56, focal weak positivity for galectin 3, and negativity for cytokeratin 19. The lesion was diagnosed as HTT based on these histological and immunohistochemical features.

Retrospectively, there were several points that could have led to the suggestion or diagnosis of HTT on FNA cytology. Ultrasonographic findings are one clue because the lesion was well circumscribed, a benign feature, although it did show hypoechoic 

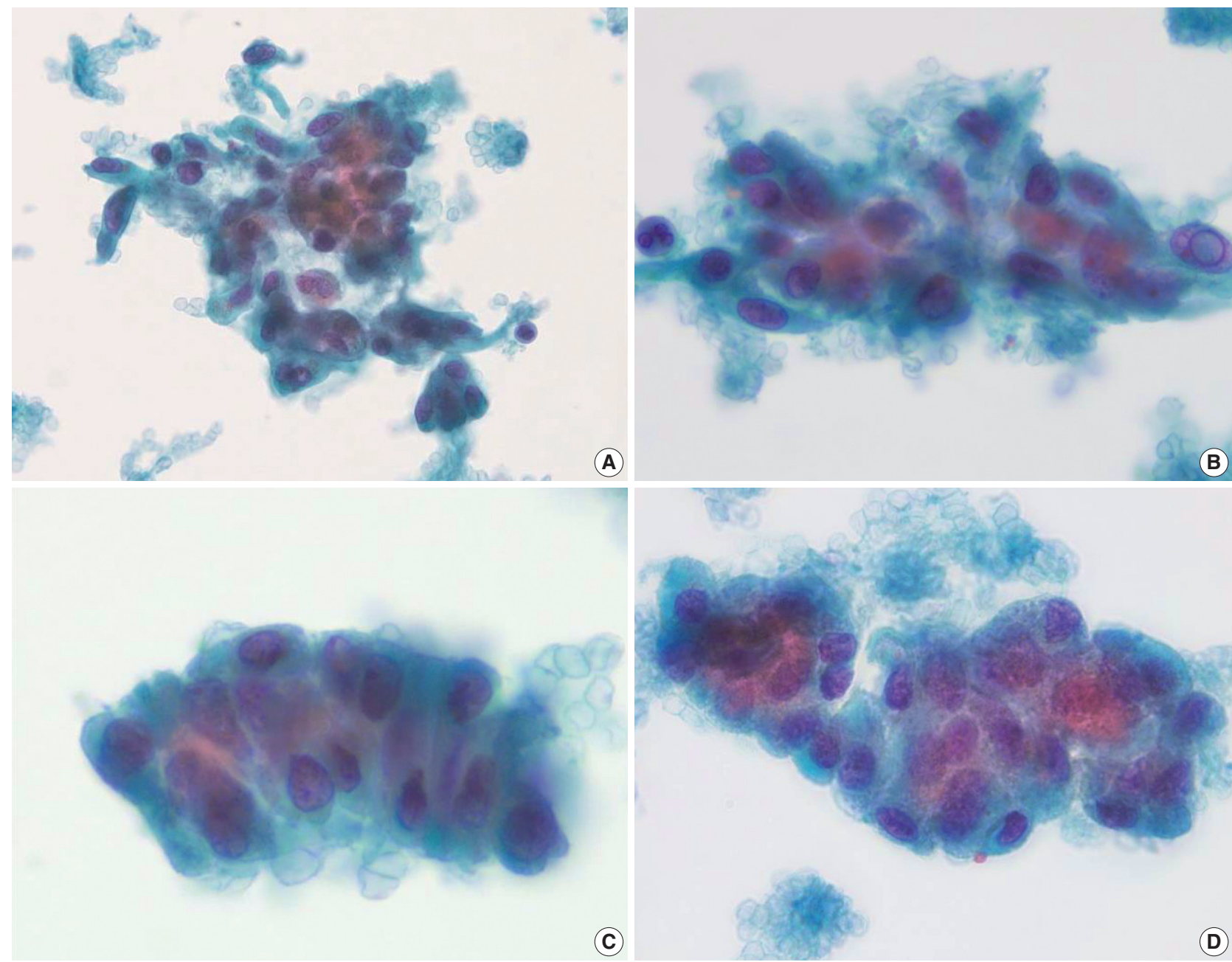

Fig. 3. Liquid based cytology (LBC; CellPrep) findings of hyalinizing trabecular tumor (HTT) (Papanicolaou). Cohesive aggregates or syncytial fragments of tumor cells around hyaline material. (A, B) Although oval to polygonal and occasionally elongated spindle-shaped tumor cells shows enlarged nuclei with hyperchromasia and occasional intranuclear pseudoinclusion as in papillary carcinoma, tumor cells in HTT shows dispersed fine chromatin rather than pale and clear chromatin, and had infrequent nuclear membrane irregularity. (C, D) Tumor cells were arranged in a more stratified trabecular pattern than in papillary carcinoma. Columnar character of tumor cells is observed in peripheral rims of clusters.

features with internal microcalcification. While the LBC of the FNA specimen had similar features to papillary carcinoma, such as occasional intranuclear pseudoinclusions and nuclear grooves, this case had some special cytological characteristics, as described below. Syncytial fragments or loosely cohesive groups of tumor cell clusters had cores of hyaline material among the cells. Tumor cells were usually oval to polygonal with pale to dense cytoplasm. However, spindle-shaped cells were occasionally observed. Unlike PTC, which has pale and clear chromatin, nuclei showed finely dispersed chromatin (Fig. 3A, B). Other than these general characteristics of HTT, more unique cytological features of this HTT case are frequent three-dimensional cell clusters and stratified columnar tumor cells in the peripheral rims of clusters.
Although tumor cells of PTC also show nuclear overlapping, HTT tumor cells in this case are more often arranged in a ball-like structure. In addition to the distinct spindle-shaped tumor cells, cells with abundant cytoplasm and eccentrically located round nuclei with columnar features were frequently observed and arranged in a stratified trabecular pattern in this case (Fig. 3C, D).

This case study was approved by the Institutional Review Board of Seegene Medical Foundation (IRB No. SMF-IRB-2018-001). The need for informed consent was waived.

\section{DISCUSSION}

HTT is a rare thyroid tumor with low malignant potential 
often misdiagnosed as PTC or MTC by FNA cytology, because of their similar cytological features. ${ }^{1,2}$ However, distinguishing characteristics of HTT must always be considered when diagnosing thyroid nodules, because diagnosis or suspicion of HTT on FNA is crucial to a correct therapeutic approach for a thyroid nodule that should not be overtreated with total or subtotal thyroidectomy. ${ }^{5}$

HTT could be considered a variant of PTC in that it has overlapping cytological features of PTC, RET/PTC rearrangement, and strong expression of galectin-3 in some cases. ${ }^{8}$ However, HTT has unique characteristics that are described below. HTT does not show BRAF (V600E) mutation. ${ }^{9}$ HTT has a unique immunohistochemical staining pattern for Ki-67 MIB1 monoclonal antibody, which is positive in peripheral tumor cell cytoplasm but expressed in the nuclei of most other tumors. ${ }^{2,10}$

Considering the characteristics of HTT mentioned above, Ki-67 immunohistochemistry of the FNA specimen, ${ }^{2}$ and RET/ PTC rearrangement, a BRAF mutation test ${ }^{9}$ would be helpful to avoid over-diagnosis of HTT as PTC or suspicious for PTC. However, immunohistochemistry and mutation tests are difficult to perform on cytology specimen and are not sufficient to rule out PTC and diagnose HTT on FNA. General use of Ki-67 staining in HTT FNA specimens is also expensive; therefore, Ki-67 staining should be limited to carefully selected inconclusive cases, for example those cases showing nuclear features of PTC but with sparse hyaline material. ${ }^{2,10,11}$

HTT should be included in the differential diagnosis of solid tumors of the thyroid gland, especially thosee showing benign US features of marked hypoechogenecity with well circumscribed smooth margin and without internal microcalcification. ${ }^{7,12-14}$

Although cytopathologists cannot directly diagnose HTT on FNA, consideration of the possibility of HTT is important to avoid misdiagnosing HTT as a malignant thyroid neoplasm such as PTC or MTC and to avoid unnecessary overtreatment for HTT, especially when intraoperative frozen section evaluation could prevent patients from undergoing overtreatment. ${ }^{14,15}$

Cytologic findings such as bloody background, low nuclear to cytoplasmic ratio, cellular aggregates around the hyaline material, fine chromatin rather than clear chromatin, and numerous nuclear grooves and intranuclear inclusions should remind pathologists of HTT as a differential diagnosis. ${ }^{4,16}$ The recognition of hyaline and colloid-like material in smears is also crucial to a correct diagnosis. ${ }^{17}$

The novel cytologic findings of our case including three-dimensional and stratified trabecular arrangement of tumor cells and columnar features of tumor cells at the periphery of cell clusters, are supportive of HTT by FNA cytology. These unique findings have never been described, especially in CellPrep LBC. Collective review of previously diagnosed HTT cases considering these unique cytologic features would be valuable to derive more exact criteria to diagnose HTT on FNA cytology.

\section{ORCID}

Soo Hee Kim: https://orcid.org/0000-0001-6715-8900

\section{Conflicts of Interest}

No potential conflict of interest relevant to this article was reported.

\section{REFERENCES}

1. Kim T, Oh YL, Kim KM, Shin JH. Diagnostic dilemmas of hyalinizing trabecular tumours on fine needle aspiration cytology: a study of seven cases with BRAF mutation analysis. Cytopathology 2011; 22: 407-13.

2. Casey MB, Sebo TJ, Carney JA. Hyalinizing trabecular adenoma of the thyroid gland identification through MIB-1 staining of fineneedle aspiration biopsy smears. Am J Clin Pathol 2004; 122: 506-10.

3. Zipkin R. Hyalinähnliche collagene Kugeln als Produkte epithelialer Zellen in malignen Strumen. Virchows Arch Pathol Anat Physiol Klin Med 1905; 182: 374-406.

4. Carney JA, Ryan J, Goellner JR. Hyalinizing trabecular adenoma of the thyroid gland. Am J Surg Pathol 1987; 11: 583-91.

5. Howard BE, Gnagi SH, Ocal IT, Hinni ML. Hyalinizing trabecular tumor masquerading as papillary thyroid carcinoma on fine-needle aspiration. ORL J Otorhinolaryngol Relat Spec 2013; 75: 309-13.

6. Jones DJ, Kieliszak CR, Patel SS, Selinsky CR. Hyalinizing trabecular tumor of the thyroid gland and its significant diagnostic issue. Thyroid Res 2017; 10: 7.

7. Saglietti C, Piana S, La Rosa S, Bongiovanni M. Hyalinizing trabecular tumour of the thyroid: fine-needle aspiration cytological diagnosis and correlation with histology. J Clin Pathol 2017; 70: 641-7.

8. Gaffney RL, Carney JA, Sebo TJ, et al. Galectin-3 expression in hyalinizing trabecular tumors of the thyroid gland. Am J Surg Pathol 2003; 27: 494-8.

9. Salvatore G, Chiappetta G, Nikiforov YE, et al. Molecular profile of hyalinizing trabecular tumours of the thyroid: high prevalence of RET/PTC rearrangements and absence of B-raf and N-ras point mutations. Eur J Cancer 2005; 41: 816-21.

10. Takada N, Hirokawa M, Ohbayashi C, et al. Re-evaluation of MIB-1 immunostaining for diagnosing hyalinizing trabecular tumour of the 
thyroid: semi-automated techniques with manual antigen retrieval are more accurate than fully automated techniques. Endocr J 2018; 65: 239-44.

11. Park HS, Kim KM, Bae JS, et al. Diagnostic caveats of immunoreactivity for Ki67 and chromogranin A in hyalinizing trabecular tumour of the thyroid. J Clin Pathol 2014; 67: 835-9.

12. Lee S, Han BK, Ko EY, Oh YL, Choe JH, Shin JH. The ultrasonography features of hyalinizing trabecular tumor of the thyroid are more consistent with its benign behavior than cytology or frozen section readings. Thyroid 2011; 21: 253-9.

13. Choi WJ, Baek JH, Ha EJ, et al. The ultrasonography features of hyalinizing trabecular tumor of the thyroid gland and the role of fine needle aspiration cytology and core needle biopsy in its diagnosis. Acta Radiol 2015; 56: 1113-8.
14. Jang $\mathrm{H}$, Park CK, Son EJ, et al. Hyalinizing trabecular tumor of the thyroid: diagnosis of a rare tumor using ultrasonography, cytology, and intraoperative frozen sections. Ultrasonography 2016; 35: 131-9.

15. Sung SY, Shen HY, Hsieh CB, et al. Hyalinizing trabecular tumor of thyroid: does frozen section prevent unnecessarily aggressive operation? Six new cases and a literature review. J Chin Med Assoc 2014; 77: 573-7.

16. Carney JA, Hirokawa M, Lloyd RV, Papotti M, Sebo TJ. Hyalinizing trabecular tumors of the thyroid gland are almost all benign. Am J Surg Pathol 2008; 32: 1877-89.

17. Bakula-Zalewska E, Cameron R, Gałczyński JP, Domanski HA. Hyaline matrix in hyalinizing trabecular tumor: findings in fineneedle aspiration smears. Diagn Cytopathol 2015; 43: 710-3. 